\title{
Reconfigurable Rateless Codes
}

Nicholas Bonello, Rong Zhang, Sheng Chen, and Lajos Hanzo

School of ECS, University of Southampton, SO17 1BJ, United Kingdom.

Email: \{nb06r,rz05r,sqc,lh\}@ecs.soton.ac.uk, http://www-mobile.ecs.soton.ac.uk

\begin{abstract}
We propose novel reconfigurable rateless codes, that are capable of not only varying the block length but also adaptively modify their encoding strategy by incrementally adjusting their degree distribution according to the prevalent channel conditions without the availability of the channel state information at the transmitter. In particular, we characterize a reconfigurable rateless code designed for the transmission of 9,500 information bits that achieves a performance, which is approximately $1 \mathrm{~dB}$ away from the discrete-input continuousoutput memoryless channel's (DCMC) capacity over a diverse range of channel signal-to-noise (SNR) ratios.
\end{abstract}

\section{INTRODUCTION}

More than a decade after the discovery of turbo codes [1] and the rediscovery of low-density parity-check (LDPC) codes [2], [3], the problem of operating arbitrarily close to capacity using practical encoding and decoding algorithms is feasible, when assuming perfect channel knowledge. These research advances were achieved with the advent of high-performance iterative decoders, and design techniques such as density evolution [4] or extrinsic information transfer (EXIT) [5] charts.

Lately, the community's interest has been shifted towards the quest for codes that are capable of maintaining this excellent performance over channels characterized with widely varying qualities within a diverse range of signal-to-noise ratios (SNR) and where the channel state information is unknown to the transmitter. By employing a conventional fixed-rate channel code over such channels, we will naturally be facing the dilemma of opting for high rates to increase the throughput or to reduce the rate in order to achieve a higher error resilience. A channel exhibiting time-variant conditions will therefore necessitate an adaptive channel coding scheme, which is exemplified by rateless (or fountain) codes, allowing us to freely vary the block length (and thus the code-rate) in order to match a wide range of fluctuating channel conditions.

Throughout this paper, we will appropriately distinguish between the instantaneous and the effective parameters using the $\overline{(\cdot)}$ notation for the former. Without delving into the intricate code-design-related details, we define what we refer to as a generic rateless encoder as an arbitrarily encoder that has the capability of generating "on-thefly" a potentially infinite bit stream from any $K$ information bits, which is denoted by the binary bit-vector $\mathbf{a}=\left(a_{1}, a_{2}, \ldots, a_{K}\right)$. Let $\bar{C}_{i}$ be a $\left(\bar{N}_{i}, K\right)$ rateless code defined over $\mathrm{GF}(2)$, which is capable of generating a codeword $\overline{\mathbf{c}_{\mathbf{i}}}=\left(c_{1}, c_{2}, \ldots, c_{\bar{N}_{i}}\right), \overline{\mathbf{c}}_{i} \in \bar{C}_{i}$, where $\bar{N}_{i}$ represents the instantaneous block length at a particular transmission instance $i$ and thus the instantaneous code-rate is defined by $\bar{R}_{i}:=K / \bar{N}_{i}$. Moreover, the code $\bar{C}_{i}$ will actually be a prefix to all succeeding codes $\bar{C}_{i+j}$ having code-rates $\bar{R}_{i+j}<\bar{R}_{i}$, for all $j>0$. A generic rateless decoder is then defined as an arbitrarily decoder, which is capable of reconstructing the original information bit sequence $\mathbf{a}$, with an arbitrarily low bit error probability from any received codeword $\mathbf{c}_{i}$ after $i$ transmission instances. The successful decision is then communicated back to the transmitter in the form of a single-bit acknowledgment (ACK) using an idealized error-free,

The work reported in this paper has formed part of the Core 4 Research Programme of the Virtual Centre of Excellence in Mobile and Personal Communications, Mobile VCE, www.mobilevce.com, whose funding support, including that of the EPSRC, is gratefully acknowledged Fully detailed technical reports on this research are available to Industrial Members of Mobile VCE. zero-delay feedback channel. Subsequently, the transmitter will cease transmission of the current information sequence $\mathbf{a}$ and proceeds to the next sequence.

It is also worth noting that some rateless code families are closely related to their fixed-rate counterparts. For instance, a Luby Transform (LT) code [6] is analogous to a non-systematic low-density generator matrix (LDGM) based code, having a generator matrix that is calculated online (and thus allowing adaptive-rate configuration for diverse channel conditions) and where the LT encoded codeword corresponds to a sequence of repeated parity-check equation values, each checking the parity of $d_{c}$ information bits. Similarly, we can regard Raptor codes [7] as a serial concatenation of a (typically) high-rate LDPC code as the outer code combined with a rateless LDGM code as the inner code.

To the best of our knowledge, the state-of-the-art rateless codes employ a fixed degree distribution [6]; i.e. the degree distribution used for coining the degree $d_{c}$ for each transmitted bit is time invariant and thus channel-independent. Consequently, such rateless codes, can only alter the number of bits transmitted in order to cater for the variations of the channel conditions encountered. A fixed-rate code having a specific rate $R$, can only attain an arbitrarily low outage probability at a particular channel condition. By the same token, rateless codes having a fixed degree distribution $\delta_{i}(x)$ are sub-optimal in the sense that they are only capable of realizing codes having rates that are arbitrarily close to the capacity for a narrow range of channel SNRs. Nevertheless, this plausible argument still suggests that having at least partial channel state information at the transmitter is still mandatory, in order to find and use the optimal degree distribution.

Motivated by this, we propose novel rateless codes, hereby referred to as reconfigurable rateless codes that are capable of not only varying the block length (and thus the rate) but also adaptively modify their encoding strategy according to the channel conditions. We will demonstrate that the proposed rateless codes are capable of shaping their own degree distribution according to near-instantaneous requirements imposed by the channel, but without the actual channel knowledge at the transmitter.

The remainder of the paper is structured as follows. Section II introduces the channel and the system model that were taken into consideration. The analysis of the proposed reconfigurable rateless codes and their adaptive incremental degree distribution is detailed in Section III. Our simulation results are then presented in Section IV, while our concluding remarks are offered in Section V.

\section{SYSTEM OVERVIEW}

\section{A. Channel model}

The canonical discrete-time complex baseband-equivalent channel model used is given by $y_{i}=h x_{i}+n_{i}$, where $x_{i}, y_{i} \in \mathbb{C}$ and $n_{i} \sim \mathcal{C N}\left(0, N_{0}\right)$ denotes the transmitted signal (i.e. the modulated codeword bit $c_{i}$ ), the received signal and the complex additive white Gaussian noise (AWGN), respectively, at any transmission instant $i$. We consider a quasi-static fading (QSF) channel having a time-invariant channel gain $h$ generated according to a complex circularly symmetric Gaussian distribution having a per-dimension noise variance of $\sigma_{n}^{2}=N_{0} / 2$. This represents a non-frequency selective channel having a coherence time $\tau$ that is higher than 


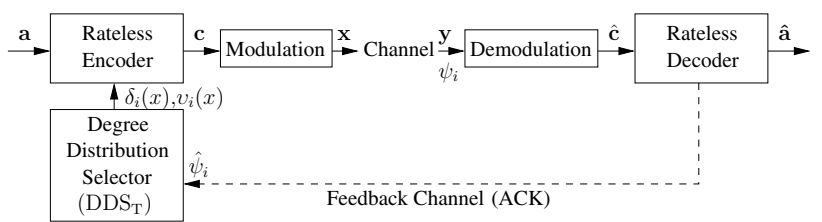

Fig. 1. The system model considered. It is also implicitly assumed that there is another subsidiary DDS located at the receiver, namely $\operatorname{DDS}_{\mathrm{R}}$, that can replicate the EXIT chart calculation and thus communicate the distributions $\delta_{i}(x)$ and $v_{i}(x)$ to the rateless decoder (please refer to Section III-A).

the system's maximum affordable codeword length determining the maximum system delay. The achievable rate supported by the arbitrarily channel gain $h$ is then defined as $C(h):=\log _{2}(1+\bar{\psi})$ bits per channel use. We note furthermore that all the attributes considered throughout this paper are computed with respect to the two-dimensional noise variance $N_{0}$ and not to $\sigma_{n}^{2}$.

The most commonly used performance metric for transmission over QSF channels is the outage probability defined as the likelihood of using an insufficiently low code-rate $\bar{R}_{i}$, which is above the channel's capacity. This is formulated as $\operatorname{Pr}_{\text {out }}\left(\bar{R}_{i}\right)=$ $\operatorname{Pr}\left(\bar{R}_{i}>C(h)\right)$, where $\bar{R}_{i}$ has the same definition given in Section I. Therefore, given a fixed-rate code of rate $R_{x}$, there exist a fading coefficient $h$ such that $\operatorname{Pr}_{\text {out }}\left(R_{x}\right)$ is non-zero. On the other hand, the outage probability $\operatorname{Pr}_{\text {out }}(R)$ of a rateless scheme may tend to zero independent of the channel conditions, since the (effective) code-rate $R$ is actually determined by the decoder (and not the encoder), when the decoding is terminated after correctly decoding a. Therefore, rateless coded transmissions over the QSF channel can be modeled as real AWGN channels having effective SNR equal of $\psi_{\text {avg. }}$.

\section{B. System Model}

The system model considered is illustrated in Figure 1. At any transmission instant $i, i=1, \ldots, N$, the rateless encoder performs the steps succinctly described in the four steps below:

1) (Degree Selection) Randomly choose a degree $d_{c}$ from a degree distribution $\delta_{i}(x)$ supplied by the degree distribution selector $\left(\mathrm{DDS}_{\mathrm{T}}\right)$;

2) (Input bit/s Selection) Randomly choose $d_{c}$ input bits from the information bit sequence $\mathbf{a}=\left(a_{1}, a_{2}, \ldots, a_{K}\right)$ having the least number of connections at the current transmission instant;

3) (Intermediate bit calculation) Calculate the value of the intermediate (check) bit $b_{i}$ by combining the $d_{c}$ input bits selected at the previous step using modulo-2 addition;

4) (Codeword bit calculation) Determine the value of the codeword bit $c_{i}$, where $c_{i}=b_{i}$ if $i=1$ or $c_{i}=b_{i} \oplus b_{i-1}$ if $i=2, \ldots, N$. The symbol $\oplus$ represents the modulo- 2 addition operation.

We also note that the complexity of this rateless encoding process described in the above steps is linear in the block length.

Continuing the analogy we have drawn between rateless and fixed-rate codes in Section I, the degree distribution $\delta_{i}(x)$ would then correspond to what is commonly referred to as the check node distribution. We will assume that all the (check) degree values of the degree distribution can represented by the vector $\mathbf{d}^{i}$, where $d_{c} \in \mathbf{d}^{i}$. Accordingly, the probability generating function $\delta_{i}(x)$ can be represented by means of a polynomial distribution given by:

$$
\begin{gathered}
\delta_{i}(x):=\sum_{\forall d_{c} \in \mathbf{d}^{i}} \delta_{d_{c}} x^{d_{c}-1}, \\
=\delta_{1}+\delta_{2} x+\ldots+\delta_{d_{c}} x^{d_{c}-1}+\ldots+\delta_{D_{c}} x^{D_{c}-1},
\end{gathered}
$$

where the positive coefficients $\delta_{d_{c}}, d_{c} \in \mathbf{d}^{i}$ denote the particular fraction of intermediate bits (or check nodes) of degree $d_{c}$ and $D_{c}=\max \left(\mathbf{d}^{i}\right)$. The variable or information node distribution can then be represented by $v_{i}(x):=x^{d_{v}^{i}-1}$, which is regular due to the second step in the encoding procedure described above.

Similarly to [6], we assume that the transmitter and the receiver have synchronized clocks used for the seed of their pseudorandom number generators, and therefore the degree $d_{c} \in \mathbf{d}^{i}$ as well as the specific modulo- 2 connections selected by both the transmitter and the receiver are identical. In order to provide further insights, below we highlight the differences between the rateless encoding technique presented above and the LT encoding method proposed by Luby ( $c f$. Section 1.1 in [6]):

1) The aim of the $\operatorname{DDS}_{T}$ is to select (or compute online) an 'appropriate' degree distribution for reconfigurable rateless codes. The $\mathrm{DDS}_{\mathrm{T}}$ is not required in the previously proposed rateless codes, such as the LT and Raptor codes, since the degree distribution is predetermined and fixed.

2) In LT codes, the $d_{c}$ information bits are selected uniformly at random, hence the actual degree $d_{v}$ attributed to each information bit can be modelled as a random variable $V$, $V \sim \pi(\lambda)$, where $\pi(\lambda)$ denotes the Poisson distribution with parameter $\lambda$. Therefore, the variable node distribution of the LT codes can be well approximated by:

$$
v_{\mathrm{LT}}(x) \approx \pi(\lambda)=\sum_{d_{v}=1}^{N} \frac{e^{-\lambda} \lambda^{d_{v}}}{d_{v} !} x^{d_{v}-1},
$$

with parameter $\lambda$ defined by $\lambda:=d_{\text {c,avg }} \frac{N}{K}$, where $K$ and $N$ are assumed to be asymptotically large. The average check node degree $d_{c, \text { avg }}$ is then given by:

$$
d_{\mathrm{c}, \text { avg }}:=\sum_{\forall d_{c} \in \mathbf{d}} \delta_{d_{c}} \cdot d_{c} .
$$

This implies that some rows of the LT code generator matrix have a low weight with a non-negligible probability, thus resulting in codes that exhibit high error floors due to their poor distance properties. Furthermore, the variable node distribution $v_{\mathrm{LT}}(x)$ represented in (2) is effectively a function of the block length, of the number of information bits as well as of the degree distribution of the LT code, $\delta_{\mathrm{LT}}(x)$. In our system, having such dependencies would have presented a problem, hence this issue will be further elaborated on in Section III-B. On the other hand, the variable node distribution $v_{i}(x)$ of the proposed rateless codes does not exhibit these dependencies.

3) The potential error floor of LDGM codes maybe mitigated by their serial concatenation with another code, which is typically another LDGM code. Motivated by this, we have added a fourth step of the rateless encoding procedure outlined at the beginning of this section, which essentially represents a unity-rate precoder (or accumulator).

In this light, the proposed codes can be considered as precoded LT codes, or instances of "rateless repeat-accumulate (RA)" codes. Establishing this relationship between fixed-rate and rateless codes will significantly simplify our forthcoming analysis, since we can conveniently model the proposed reconfigurable rateless codes as non-systematic RA codes.

\section{System DESCRIPTION}

Up to this point in time, the $\operatorname{DDS}_{\mathrm{T}}$ of Figure 1 was treated as a black box capable of calculating the degree distribution $\delta_{i}(x)$ online 


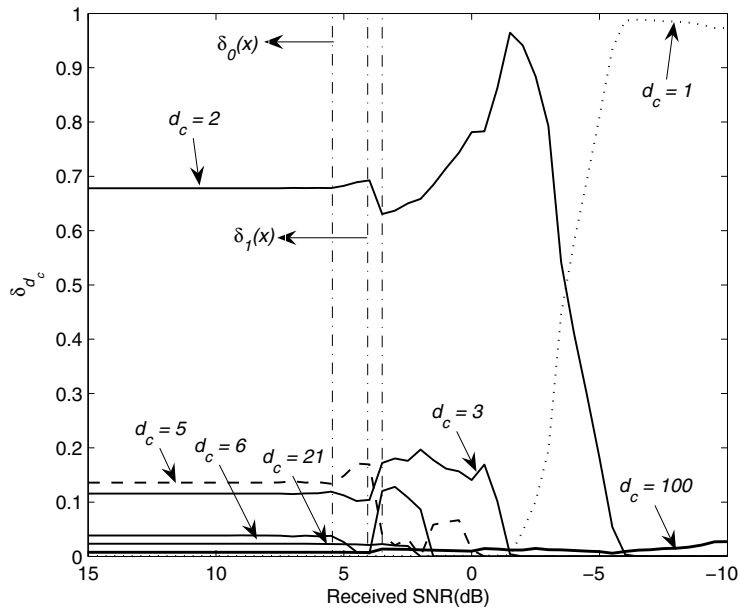

Fig. 2. The fraction of check nodes of degree $d_{c} \in \mathbf{d}^{i}, \delta_{d_{c}}$, with $i \geq 0$, calculated by the $\operatorname{DDS}_{\mathrm{T}}$ of Figure 1 under the assumptions detailed in Section III-A.

by observing the feedback channel's output. In Section III-A, we will simplify our analysis by temporarily assuming that the $\mathrm{DDS}_{\mathrm{T}}$ of Figure 1 is equipped with perfect channel knowledge and thus is capable of determining the optimal degree distribution that facilitates a near-capacity performance. This assumption is then discarded in Section III-A, where we only assume having perfect channel state information at the receiver.

\section{A. Analysis under simplified assumptions}

In this sub-section we will stipulate the following simplifying assumptions: (a) perfect channel knowledge is available at both the receiver as well as at the transmitter; (b) the rateless decoder is not bounded in terms of its complexity; (c) there exists an SNR for the rateless code $C$ above which an arbitrarily small BER/BLER probability can be achieved in the limit for asymptotically large block lengths; and (d) the decoder is capable of detecting whether the decoded $\hat{\mathbf{a}}=\mathbf{a}$.

Using the fixed-rate versus rateless code analogy introduced in the previous sections, the rateless decoder of Figure 1 is constituted of two decoders separated by a uniform random interleaver, where the inner decoder is the amalgam of a memory-one trellis decoder used for the accumulator and of a check node decoder (CND), whilst the outer decoder is a variable node decoder (VND). We will assume that the interleavers have sufficiently high girth to ensure that the non-negligible correlations between the extrinsic log-likelihood ratios do not have a severe impact on the decoder.

The convergence behavior of this iterative rateless decoding process can then be analyzed in terms of the evolution of the input and output mutual information exchange between the inner and outer decoders in consecutive iterations, which is diagrammatically represented using the semi-analytical tool of EXIT charts [5]. There are three requirements to be satisfied in order to design a nearcapacity system; (a) both the inner as well as the outer decoder's EXIT curves should reach the $(1,1)$ point on the EXIT chart; (b) the inner decoder's curve $I_{A C C \& C N D}$ should always be above the outer decoder's curve $I_{V N D}$ and (c) the $I_{A C C \& C N D}$ curve has to match the shape of the the $I_{V N D}$ curve as accurately as possible, thus resulting in an infinitesimally low EXIT-chart-tunnel area.

Given the distributions $v_{i}(x)$ and $\delta_{i}(x)$, the two EXIT curves correspond to two EXIT functions formulated by [5]:

$$
I_{E, V N D}\left(I_{A, V N D}, d_{v}^{i}\right)=J\left(\sqrt{\left(d_{v}^{i}-1\right)} \cdot J^{-1}\left(I_{A, V N D}\right)\right),
$$

where the function $J(\cdot)$ denotes the mutual information, $I_{E, V N D}\left(I_{A, V N D}, d_{v}^{i}\right)$ represents the extrinsic information output of the VND as a function of the its a-priori information input $I_{A, V N D}$ and its variable node degree $d_{v}^{i}$. Similarly, the combined accumulator and CND EXIT function $I_{E, A C C \& C N D}(\cdot)$ is then approximated by [5]:

$$
\begin{aligned}
& I_{E, A C C \& C N D}\left(I_{A, C N D}, \mathbf{d}^{i}, \psi_{\mathrm{avg}}\right) \approx \sum_{\forall d_{c} \in \mathbf{d}^{i}} \Delta_{d_{c}}^{i}[1- \\
& \left.J\left(\sqrt{\left(d_{c}-1\right) \cdot\left[J^{-1}\left(1-I_{A}\right]^{2}+\left[J^{-1}\left(1-I_{E}\right)\right]^{2}\right.}\right)\right],
\end{aligned}
$$

where $I_{A}:=I_{A, C N D}$ represents the a-priori information input of the CND and the extrinsic information accumulator output is defined by $I_{E}:=I_{E, A C C}\left(I_{A, A C C}\right)$, where $I_{A, A C C}$ denotes the a-priori accumulator information input. The parameter $\Delta_{d_{c}}^{i}$ corresponds to the specific fraction of edges emanating from the intermediate bits (or check nodes) of degree $d_{c} \in \mathbf{d}^{i}$ given by $\Delta_{d_{c}}^{i}=\delta_{d_{c}} \cdot \frac{d_{c}}{d_{c} \text { avg }}$ and the average check node degree $d_{\mathrm{c} \text {,avg }}$ is defined in (3). Furthermore, we note that designing the two EXIT curves determines the two distributions and vice versa.

Consider the scenario of having binary phase-shift keying (BPSK) modulation transmissions over the BIAWGN channel characterized by SNRs ranging from -10 to $15 \mathrm{~dB}$. If the $\mathrm{DDS}_{\mathrm{T}}$ of Figure 1 possesses perfect channel knowledge, then it is capable of computing online the decoder's EXIT curves that satisfy the above three requirements, and from which we can determine the distributions $\delta_{i}(x)$ and $v_{i}(x)$. The result of this experiment is portrayed in Figure 2, which shows particular fraction of check nodes of degree $d_{c}, \delta_{d_{c}}$, that characterize the degree distribution $\delta_{i}(x)$ across the range of SNR values considered. It can be observed from Figure 2 that the characteristics of the degree distribution $\delta_{i}(x)$ across this range of SNRs are so distinctively dissimilar, which also highlights the inadequacy of a rateless codes having a fixed degree distribution in the face of time-variant SNRs. For example, the check degrees $d_{c}>2$ are the dominant degrees at high channel SNR values, whilst they are almost extinct when the channel quality is poor. Furthermore, we note that at low channel SNR values, the system reduces to a simple repetition code, with the exception of a very small percentage of nodes having $d_{c}=100$.

We emphasize that a non-systematic rateless coding scheme was preferred over its systematic counterpart in order to completely eliminate the dependency of the variable node distribution on the channel condition. This can also be verified from (4). By doing so, the channel dependency has been confined to only one of the two distributions; i.e. to $\delta_{i}(x)$ corresponding to the $I_{A C C \& C N D}$ EXIT curve. However, the outer decoder's EXIT curve $I_{V N D}$ will now emerge from the $(0,0)$ point of the EXIT chart and hence a certain percentage of degree-one check nodes $\delta_{d_{1}}$ is always required in order to force the $I_{A C C \& C N D}$ curve to emerge from a higher initial value than the $I_{V N D}$ curve and thus guarantee that the iterative decoder begins to converge. This percentage of doped check nodes $\delta_{d_{1}}$ is also dependent on the channel quality, but the optimal $I_{A C C \& C N D}$ curve is channel-quality dependent anyway.

\section{B. The adaptive incremental degree distribution}

In this subsection, we will no longer assume perfect channel state information at the transmitter, but only a single-bit ACK transmitted by the receiver on the feedback channel in a similar fashion to that used in incremental redundancy aided schemes. We were particularly interested in finding the answer as to whether it is possible to design a variable incremental degree distribution, that attempts to imitate the attributes of the optimal channel-state 
dependent one. From another point of view, this question can be restated as to whether it is possible for the $\operatorname{DDS}_{\mathrm{T}}$ to estimate the inner decoder's EXIT curve $I_{A C C \& C N D}$, so that near-capacity performance is guaranteed, regardless of the channel conditions encountered. Once the $I_{A C C \& C N D}$ EXIT curve is computed, the degree distribution $\delta_{i}(x)$ can be readily calculated and passed on to the rateless encoder. Hence there is a need for encoders having the capability of "thinking like decoders" before encoding.

Against this backdrop, we introduce what we refer to as the adaptive incremental distribution. The $\mathrm{DDS}_{\mathrm{T}}$ of Figure 1 is initialized by making a conjecture of the channel quality. For example, the initial estimate $\hat{\psi}_{0}$ provided for the DDS ${ }_{\mathrm{T}}$ of Figure 1 can be set to the highest SNR considered, i.e. $15 \mathrm{~dB}$, in an attempt to maximize the achievable throughput. However, it can be observed from Figure 2 that the rateless decoder should still be able to successfully decode $\hat{\mathbf{a}}=\mathbf{a}$ using the same distribution $\delta_{0}(x)$, even if the receiver experiences an SNR as low as $5 \mathrm{~dB}$. Therefore, the estimate $\hat{\psi}_{0}$ can be set to the latter value. Then, the rateless encoder employs the degree distribution $\delta_{0}(x)$ designed for a code having a rate of 0.9681 , which is given by $\delta_{0}(x)=0.0007+0.6781 x+$ $0.1156 x^{2}+0.1358 x^{4}+0.0386 x^{5}+0.0235 x^{20}+0.0077 x^{99}$ and $v_{0}(x)=x^{3}$.

The $\mathrm{DDS}_{\mathrm{T}}$ is continuously observing the feedback channel output and must try its utmost to glean as much information as possible from it. While it is plausible that the simple ACK feedback is less beneficial than having complete channel knowledge, the ACK as well as the absence of the ACK can still prove to be useful for the $\mathrm{DDS}_{\mathrm{T}}$ to improve the estimate of $\hat{\psi}_{0}$. Recall from Section III-A, that if $\operatorname{DDS}_{\mathrm{T}}$ posses a precise estimate of the channel quality, then the problem is basically solved since the $\mathrm{DDS}_{\mathrm{T}}$ is capable for calculating the specific degree distribution that achieves a performance arbitrarily close to capacity.

To elaborate further, it can be argued that the absence of a received ACK may indicate two options for the $\mathrm{DDS}_{\mathrm{T}}$; either that the estimate of $\hat{\psi}_{0}$ is correct but the rateless decoder is unsuccessful in correctly decoding a due to using an insufficient number of iterations or $\hat{\psi}_{0}$ is representing an overly optimistic estimate of the channel conditions. We note that the first possibility must not to be completely neglected, especially when considering that the EXIT curves corresponding to the two distributions are closely matched in an attempt to maximize the achievable throughput and therefore a considerable number of iterations is necessary. If this occurs, then transmitting some additional redundant bits may make up for the limited number of affordable iterations. Thus we pay a rate-penalty in exchange for a lower computational complexity. On the other hand, if the $\mathrm{DDS}_{\mathrm{T}}$ has an incorrect estimate of the channel condition and thus no ACK has been received, two further possibilities might have occurred. Namely, the rateless decoder may have either started the decoding but was unsuccessful or else it did not even attempt to decode the received codeword, because $\bar{R}<C(h)$.

Since the SNR range considered is quite wide, we assume that the most likely cause of failure is feeding the $\mathrm{DDS}_{\mathrm{T}}$ with an inaccurate $\hat{\psi}_{0}$ and so, a modification of the encoding strategy (thus a modification of the degree distribution $\delta_{0}(x)$ and $v_{0}(x)$ ) is required. Therefore, if an ACK is still not received after transmitting according to the degree distribution $\delta_{0}(x)$, then the $\mathrm{DDS}_{\mathrm{T}}$ of Figure 1 can reasonably assume that its next estimate is $\hat{\psi}_{1} \leq 5 \mathrm{~dB}$ (please refer to Figure 2). The immediate problem that has to be tackled by the $\mathrm{DDS}_{\mathrm{T}}$ is that of calculating an improved degree distribution $\delta_{1}(x)$ for the improved estimate $\hat{\psi}_{1}$, given that the previous distribution was $\delta_{0}(x)$. This can be viewed as an optimization problem, i.e. given that having an unsuccessful $\delta_{i}(x)$ was attributed to the inaccurate channel quality estimate $\hat{\psi}_{i}$, the next degree distribution $\delta_{i+1}(x)$ can be determined by:

$$
\delta_{i+1}(x)=\max \sum_{\forall d_{c} \in \mathbf{d}^{i+1}} \frac{d_{c}}{\Delta_{d_{c}}^{i+1}}
$$

subject to the equality constraint

$$
\sum_{\forall d_{c} \in \mathbf{d}^{i+1}} \Delta_{d_{c}}^{i+1}=1
$$

and to the inequality constraints given by

$$
I_{E, A C C \& C N D}\left(\mathcal{I}, \mathbf{d}^{i+1}, \hat{\psi}_{i+1}\right)>I_{A, V N D}\left(\mathcal{I}, d_{v}^{i+1}\right),
$$

and

$$
\left.\Delta_{d_{c}}^{i+1}\right|_{\forall d_{c} \in\left(\mathbf{d}^{i+1} \backslash \mathbf{d}^{i}\right)}>0,
$$

where $\mathbf{d}^{i+1}$ is the vector containing all the parity-check degree values of the next degree distribution $\delta_{i+1}(x), \mathbf{d}^{i} \subseteq \mathbf{d}^{i+1}$, and $\hat{\psi}_{i+1}<\hat{\psi}_{i}$ is the new channel quality estimate. In $(8), \mathcal{I}$ is a discrete set of gradually increasing values in the interval $[0,1]$ over which the functions $I_{E, A C C \& C N D}(\cdot)$ and $I_{A, V N D}(\cdot)=I_{E, V N D}^{-1}(\cdot)$ (please refer to (5) and (4)) are calculated. The specific value of $d_{v}^{i+1}$ is selected by considering the smallest variable node degree value that satisfies both $d_{v}^{i+1}>d_{v}^{i}$ as well as (8). We further note that the maximization of the objective function in (6) is equivalent to maximizing the code-rate.

An important step to consider is that the newly calculated degree distribution $\delta_{i+1}(x)$ must take into account the previous $\delta_{i}(x)$, since the bits connected to the degrees $d_{c} \in \mathbf{d}^{i}$ coined from $\delta_{i}(x)$ have already been transmitted and thus will still affect the rateless decoding. Due to this, we introduce an additional inequality constraint, in addition to that given by (8) and (9), expressed by:

$$
\left.\Delta_{d_{c}}^{i+1}\right|_{\forall d_{c} \in\left(\mathbf{d}^{i} \cap \mathbf{d}^{i+1}\right)} \geq \frac{d_{\mathrm{v}, \text { avg }}^{i}}{d_{\mathrm{v}, \text { avg }}^{i+1}} \cdot \Delta_{d_{c}}^{i} .
$$

The adaptive incremental distribution denoted by $\delta_{\text {adap }}(x, \hat{\psi})$ employed by the proposed reconfigurable rateless codes instead of a fixed one can be formulated as:

$$
\begin{array}{r}
\delta_{\text {adap }}(x, \hat{\psi}):=\delta_{0}(x) \mathbf{1}\left\{\hat{\psi} \geq \hat{\psi}_{0}\right\}+\delta_{1}(x) \mathbf{1}\left\{\hat{\psi}_{0}>\hat{\psi} \geq \hat{\psi}_{1}\right\} \\
+\ldots+\delta_{z}(x) \mathbf{1}\left\{\hat{\psi}_{z-1}>\hat{\psi} \geq \hat{\psi}_{z}\right\}
\end{array}
$$

where the $\operatorname{DDS}_{\mathrm{T}}$ channel quality estimate is $\hat{\psi} \in\left(\hat{\psi}_{0}, \hat{\psi}_{1}, \ldots, \hat{\psi}_{z}\right)$ and where $\mathbf{1}\{\cdot\}$ denotes the indicator function returning a value of one, if the argument is true, and zero otherwise. As a further example, the next incremental distribution $\delta_{1}(x)$ (and $v_{1}(x)$ ) determined by relying on the distribution $\delta_{0}(x)$, is calculated by solving the linear programming problem outlined in (6)-(10), which leads to $\delta_{1}(x)=0.0010+0.6400 x+0.1375 x^{2}+0.1281 x^{4}+0.0364 x^{5}+$ $0.0188 x^{7}+0.0023 x^{8}+0.0221 x^{20}+0.0138 x^{99}$ and $v_{1}(x)=x^{4}$.

\section{Simulation Results}

We compared our results to both Raptor codes as well as to punctured regular and irregular LDPC codes. The Raptor code [7] was constructed by serially concatenating a regular LDPC outer code described by a PCM having a column weight of 3 and a row weight of 30 and thus realizing a rate- 0.9 code. This LDPC code was then concatenated with a non-systematic LT code having a fixed degree distribution given by $\delta_{\mathrm{LT}}(x)=0.05 x+0.5 x^{2}+$ $0.05 x^{3}+0.25 x^{4}+0.05 x^{6}+0.1 x^{8}$. On the other hand, the proposed reconfigurable rateless codes employ an adaptive incremental degree distribution $\delta_{\text {adap }}(x, \hat{\psi})$ represented in (11), which were initialized with the distributions $\delta_{0}(x)$ and $v_{0}(x)$. The number of information 


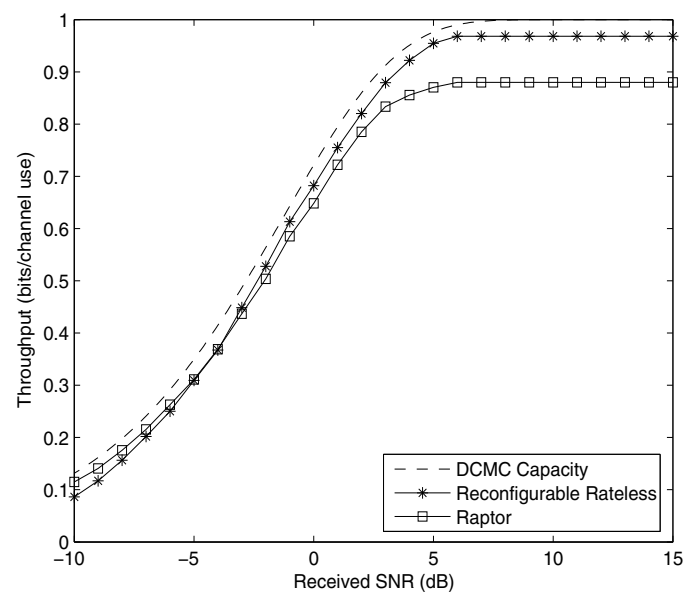

(a) Reconfigurable rateless vs Raptor (BPSK, QSF channel)

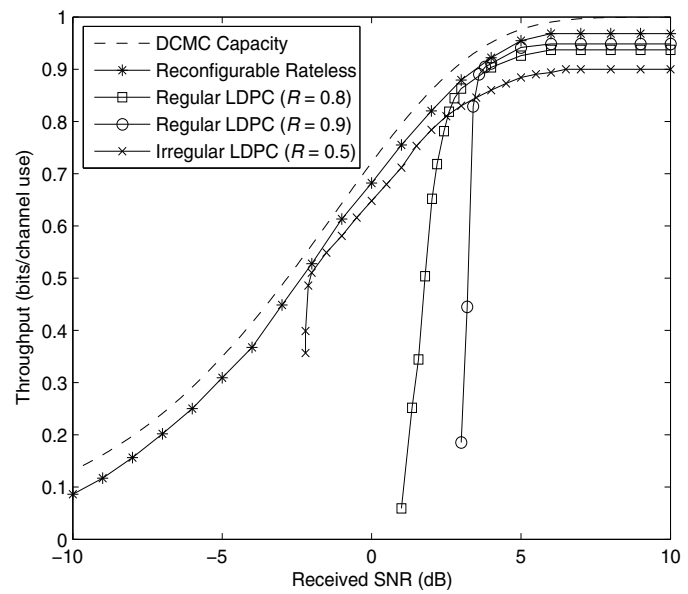

(b) Reconfigurable rateless vs punctured LDPC (BPSK, BIAWGN channel)

Fig. 3. Average throughput (bits/channel use) versus SNR (dB). The rateless decoder was limited to a maximum of 200 iterations.

bits $K$ to be recovered was set to 9,500 bits and the incremental redundancy segment used for both schemes was set to 100 bits.

Figure 3(a) illustrates the exhibited average throughput performance versus the SNR for the proposed reconfigurable rateless codes. It can be observed that the proposed codes achieve a performance within approximately $1 \mathrm{~dB}$ of the discrete-input continuous-output memoryless channel's (DCMC) capacity across a diverse range of SNRs. Furthermore, it can be verified that the performance exhibited by the reconfigurable rateless codes is superior to that of the Raptor code for all SNRs higher than -4 $\mathrm{dB}$. For example at $-3 \mathrm{~dB}$ and $0 \mathrm{~dB}$, the proposed codes require on average 560 and 730 less redundant bits than the corresponding Raptor benchmarker code. On the other hand, Raptor codes excel at low SNR, and are suitable candidates to be used for signaling when the channel quality may become very poor [8]

The excellent performance exhibited by the proposed rateless reconfigurable codes at medium-to-high SNRs can be explained by their optimistic philosophy in calculating the channel quality estimate. The higher the average received SNR, the faster it is for the $\mathrm{DDS}_{\mathrm{T}}$ to estimate the channel quality and the more accurate the adaptive incremental degree distribution becomes. The effect is actually reversed, when the received SNR is very low, since the adaptive incremental degree distribution $\delta_{\text {adap }}(x, \hat{\psi})=\delta_{z}(x)$ employed in this case is still taking into effect the previous distributions $\delta_{y}(x)$, for all $0 \geq y<z$, that were used to transmit a fraction of $N$ bits, when the $\operatorname{DDS}_{\mathrm{T}}$ had an optimistic channel quality estimate $\hat{\psi}_{y}$.

Soljanin et al. in [8] demonstrated that in the high-SNR region, the performance exhibited by punctured LDPC codes is superior to that of Raptor codes. Therefore, it was of interest to verify, whether the performance of punctured LDPC codes is also superior to that exhibited by the proposed rateless reconfigurable codes. We have considered the same scenario as in [8], i.e. used a high-rate regular LDPC code such as the previously described rate-0.9 outer LDPC code employed for the Raptor code as well a rate-0.8 LDPC code having a PCM of column-weight 3 and row-weight 15 . We also considered a half-rate irregular LDPC code having a variable node distribution given by $v(x)=$ $0.2199 x+0.2333 x^{2}+0.0206 x^{3}+0.0854 x^{4}+0.0654 x^{6}+0.0477 x^{7}+$ $0.0191 x^{8}+0.0806 x^{18}+0.2280 x^{19}$ and a check node distribution represented by $\delta(x)=0.6485 x^{7}+0.3475 x^{8}+0.0040 x^{9}$, where both distributions were optimized using density evolution [4]. Our performance comparison in terms of the average throughput (bits/channel use) versus SNR (dB) over the BIAWGN channel between the proposed reconfigurable rateless codes as well as the incremental-redundancy-based HARQ schemes using punctured regular and irregular LDPCs is illustrated in Figure 3(b). It is demonstrated in Figure 3(b), that the performance of the proposed rateless reconfigurable codes is also superior to that of punctured regular and irregular LDPC codes.

\section{SUMMARY AND CONCLUDING REMARKS}

In this paper, we have proposed novel reconfigurable rateless codes, that are capable of not only varying the block length but also shape their own degree distribution according to requirements imposed by the channel and without the availability of the channel state information at the transmitter. Our method is reminiscent of what is referred to as EXIT chart matching, however it is now applied in the context of rateless codes and therefore must also be performed "on-the-fly". A reconfigurable rateless code was characterized for transmission of 9500 information bits over quasistatic channels and achieves a performance that is approximately 1 $\mathrm{dB}$ away from the DCMC capacity over a diverse range of channel signal-to-noise (SNR) ratios.

\section{REFERENCES}

[1] C. Berrou, A. Glavieux, and P. Thitimajshima, "Near Shannon limi error-correcting coding and decoding: Turbo-codes," in Proceedings of the International Conference on Communications, Geneva Technical Program, vol. 2, (Geneva, Switzerland), pp. 1064-1070, May 23-26, 1993.

[2] R. G. Gallager, "Low-density parity-check codes" IRE Transaction Information Theory, vol. 45, pp. 21-28, Jan. 1962.

[3] D. MacKay, "Good error-correcting codes based on very sparse matrices," IEEE Transactions on Information Theory, vol. 45, pp. 399 431, Mar. 1999.

[4] T. J. Richardson and R. Urbanke, "Design of capacity-approaching irregular low-density parity-check codes," IEEE Transactions on Information Theory, vol. 47, pp. 619-637, Feb. 2001.

[5] S. ten Brink and G. Kramer, "Design of repeat-accumulate codes for iterative detection and decoding," IEEE Transactions on Signal Processing, vol. 51, pp. 2764-2772, Nov. 2003.

[6] M. Luby, "LT codes," in Proceedings of 43rd Annual IEEE Symposium on Foundations of Computer Science, pp. 271-280, Nov. 16-19, 2002.

[7] A. Shokrollahi, "Raptor codes," IEEE Transactions on Information Theory, vol. 52, pp. 2551-2567, June 2006.

[8] E. Soljanin, N. Varnica, and P. Whiting, "Punctured vs rateless codes for hybrid ARQ," in Proceedings of IEEE Information Theory Workshop, (Punta del Este, Uruguay), pp. 155-159, 2006. 